\title{
Investigation of Short-term Amplitude and Frequency Fluctuations of Lasers for Interferometry
}

\author{
J. Hrabina, J. Lazar, M. Holá, O. Č́p \\ Institute of Scientific Instruments, v.v.i., Academy of Sciences of the Czech Republic, \\ Královopolská 147, 61264 Brno, Czech Republic, hrabina@isibrno.cz
}

\begin{abstract}
One of the limiting factors of accuracy and resolution in laser interferometry is represented by noise properties of the laser powering the interferometer. Amplitude and especially frequency fluctuations of the laser source are crucial in precision distance measurement. Sufficiently high long-term frequency stability of the laser source must be achieved especially in applications in fundamental metrology. Furthermore, the short-term frequency variations are also important primarily for measurements done at high acquisition speeds. This contribution presents practical results of measurements of short-term amplitude and frequency noises of a set of laser sources commonly used in laser interferometry. The influence of the interferometer design and electrical parameters of the detection system are also discussed.
\end{abstract}

Keywords: Nanometrology, laser noise, AFM, spectroscopy, interferometry

\section{INTRODUCTION}

L ASER INTERFEROMETRY is the key technique in metrology of length. It is able to cover measurement ranges from kilometers in long-distance interferometers to subnanometer distances in the most precise laboratory machines like interferometric comparators, nanometrology systems and metrological CMMs (coordinate measurement machines [1-8]). In case of fundamental metrology laboratory devices it is also crucial that the laser as a source for powering the interferometer directly fulfills the demand for traceability to the primary standard of length - it follows one of the recommendations for realization of the meter standard [9]. One of the most important parameters is longterm and also short-term frequency stability of the laser source. As the interferometer uses the wavelength of the laser as the "rule", every frequency fluctuation affects the measurement. Suitable laser source for interferometric systems should have minimal amplitude and also frequency noise which can influence accuracy, resolution and finally the uncertainty of the whole measurement, and in case of multidimensional interferometric devices it should also be able to offer sufficient power level for powering all interferometers incorporated in the system. We concentrated our work towards investigation of short-term frequency fluctuations, especially with the focus on applications in metrological interferometry systems.

\section{FREQUENCY STABILITY}

One of the most crucial preconditions for correct operation of measuring systems for high-precision interferometry, for example, in nanometrology is to have sufficient frequency stability of the laser source which powers the interferometers. Commercial interferometers usually rely on He-Ne laser which offers visible laser light, low price and relatively good intrinsic long-term frequency stability [1015]. The main advantages of frequency doubled Nd:YAG lasers in comparison to He-Ne are: more laser power needed for supplying multiaxis coordinate interferometers, slightly shorter wavelength which means better resolution of the measured distances, and also there are stronger and narrower iodine absorption lines in the $532 \mathrm{~nm}$ spectral region which allow easier stabilization schemes using, e.g., linear absorption spectroscopy technique for locking of the laser optical frequency to a selected transition [16-19]. The relative frequency stability of DPSSL (diode pumped solid state laser) monolithic Nd:YAG lasers stabilized by this technique was measured in range of $4 \times 10^{-9}$ for an integration time of $100 \mathrm{~s}$ and measurements were performed on air conditions (frequency modulation of the laser was done through slow thermal tuning only) [20-22]. This frequency stability is fully sufficient for measurements that are done on air conditions - the influence of the refractive index of air fluctuations is in the order of $10^{-7}$ or can be compensated up to this level [23-31]. Next, the relative frequency stability of frequency doubled Nd:YAGs stabilized by some more sophisticated technique like saturated subdoppler spectroscopy in iodine vapor can be in the range close to the $10^{-14}$ level for $100 \mathrm{~s}$ integration times [32-38]. Furthermore, not only long-term but also short-term frequency stability is important, especially in cases of high-speed interferometric systems. For example, metrological AFM tools often employ fast scanning-mode measurements, where a large amount of samples of microscope probe position must be measured at high acquisition speeds. The computing speed also plays a significant role in multidimensional and fastmoving devices. So when the measurement must be done quickly, the short-term fluctuations can be the key limiting factor of accuracy and uncertainty.

\section{RESOLUTION OF THE INTERFEROMETER AND DETECTION}

With digital signal processing in the detection chain the resolution of the interferometer may be seen as a resolution of A/D conversion (no say about the wavelength) from the technical point of view. Of course with a high-resolution $\mathrm{A} / \mathrm{D}$ converter, not only the least significant bit may be sunk in noise. Larger difference between the interferometer beam paths (reference and measuring) mean higher sensitivity of the interferometer to frequency noise of the laser source. 
The variations of wavelength can be seen as a source of multiplicative error which contributes to the absolute deviations from the measured value more on a larger distance. The next important factor, especially in case of a need for high-speed measurement is bandwidth of the whole interferometric system [39]. Broader bandwidth lets a higher portion of the noise spectral density in. Namely in case of coordinate multidimensional scanning measurement systems the acquisition speed is crucial. Also processing and evaluation of the interference signal including electronic hardware and software (demodulation and linearization techniques) must be considered during the interferometer design process [40-47]. Each component plays a role in the evaluation of the overall uncertainty of the interferometric systems.

\section{SHORT-TERM FREQUENCY NOISE EXPERIMENT}

We set up an experimental arrangement for simultaneous measuring of both amplitude and short-term frequency noise of several single-frequency $\mathrm{He}-\mathrm{Ne}$ and also frequency doubled Nd:YAG lasers. The optical power amplitude fluctuations were measured directly by a photodetector, frequency noise of the laser radiation was measured with the help of a passive Fabry-Perot cavity used as an optical frequency discriminator. This cavity contained a mirror holder equipped with piezoelectric actuator (PZT) which allowed frequency tuning of the cavity length. The length of tunable Fabry-Perot cavity was in the next step stabilized by slow servo-loop $(\tau \sim 3$ s) to the investigated laser optical frequency, so the frequency of the laser matched the middle point of the slope of the resonant transmission curve of the cavity. In this regime the cavity operated as a frequency discriminator suitable for fast laser noise fluctuation measurement. The free spectral range of the cavity was 2 $\mathrm{GHz}$ and measured linewidth of the cavity was $25 \mathrm{MHz}$. While the output signal of this frequency discriminator contained both, amplitude and frequency noises, the amplitude noise measured directly by the photodetector and frequency fluctuations investigated through the cavity were recorded simultaneously. This gave us an opportunity to subtract the influence of amplitude noise from the frequency discriminator output. The cavity was inserted into a thermalshielded chamber to suppress the influences of fluctuating refractive index of air.

The opportunity to compare the results of these noise measurements through the Fabry-Perot cavity with the performance of a length measuring interferometer under the presence of laser noise was achieved by adding another part to the measurement setup - a Michelson interferometer with homodyne quadrature detection. The interferometer reacts to variations of the input laser frequency by changes of the phase of the signal on the output of the quadrature detection unit so it can be seen as another kind of a frequency discriminator operating within the range of a single interference fringe. The key advantage of this approach is only negligible influence of amplitude fluctuations of the laser power to the output signal because the detection system response to these fluctuations is a few times smaller in comparison to frequency noise. We used the Michelson interferometer in four-pass configuration and a flat mirror reflector. A schematic of the interferometer configuration is in Fig.2. The length difference between reference and measurement paths of the interferometer was $2 \mathrm{~m}(500 \mathrm{~mm}$ mechanical distance).

All of the photodetectors were based on FDS010 photodiodes from Thorlabs, with NEP $=5 * 10^{-14} \mathrm{~W} / \mathrm{Hz}^{(1 / 2)}$ (noise equivalent power) and dark current at the level of 2 nA together with ultra-low noise operational amplifiers (OP97 from Analog Devices) that keep their noise background below the laser noise level.

Both measurements (with the help of the Fabry-Perot cavity and also with the help of interferometer) were done in the same time to have a possibility of comparison of the results. Measured signals were simultaneously recorded with the digital acquisition card with the 16 bit resolution and $600 \mathrm{kSamples} / \mathrm{s}$ per channel sampling. The whole experimental setup schematic is in Fig.1.

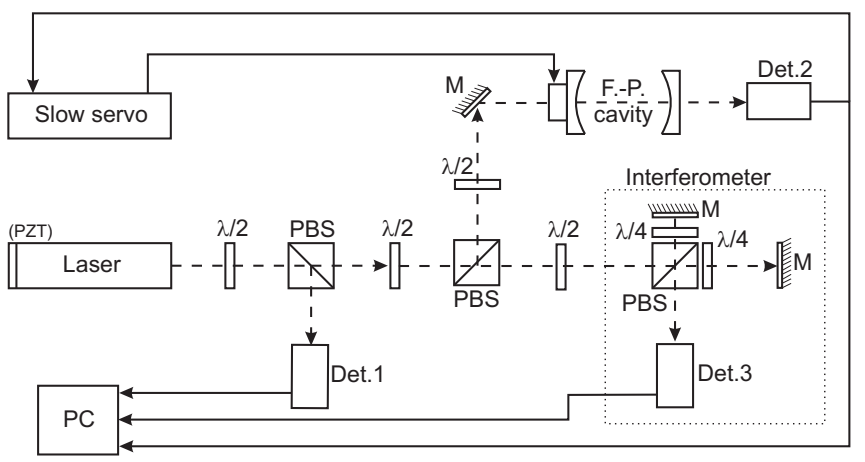

Fig.1. The principal schematic of measurement setup for amplitude and frequency properties of the tested laser source investigation. PBS-polarizing beam splitters, M-mirrors, Det-photodetectors. The PZT of the L1 laser was used for controlled frequency modulation during the evaluation of correct work of both frequency discriminators.

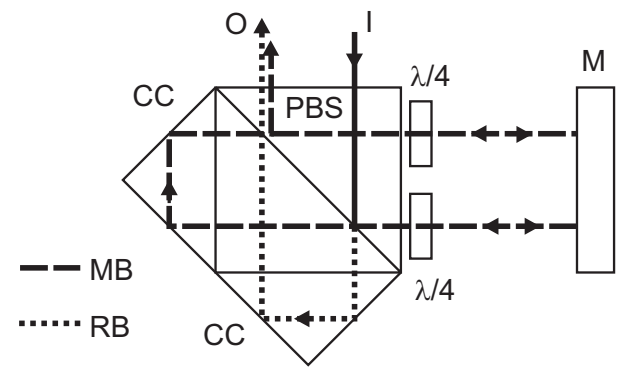

Fig.2. The schematic of used four-pass Michelson interferometer. MB-measuring beam, RB-reference beam, CC-corner cubes, PBSpolarizing beam splitter, $\mathrm{M}$-measuring mirror, I-input, O-output of the beam.

\section{EXPERIMENTAL RESULTS}

The amplitude and frequency noise were measured for different laser sources (Table 1.). All of the lasers were tested in free-running regime. However, L5 laser head contained thermal prestabilization by 2 -mode technique which could not be switched off. The amplitude noise recordings are referenced to optical power of $1 \mathrm{~mW}(0 \mathrm{~dB}=$ $1 \mathrm{~mW})$, the frequency noise recordings are referenced to $1 \mathrm{MHz}$ frequency drift $(0 \mathrm{~dB}=1 \mathrm{MHz})$. 
L1 (L2) laser is ultra-stable ring Nd:YAG intended for metrology applications. It offers the "noise-eater" option which means switchable internal filter of amplitude noise. Measurement with activated and also deactivated filter was done (signed as L1 and L2, respectively). Denoted spectral linewidth is $1 \mathrm{kHz}$ level $(0.1 \mathrm{~s})$, intensity noise without noise-eater option is below $0.1 \%(10 \mathrm{~Hz}-2 \mathrm{MHz})$, and below $0.06 \%(10 \mathrm{~Hz}-2 \mathrm{MHz})$ with activated noise-eater filter. L3 laser is ultra-stable diode-pumped ring laser with an intracavity frequency doubling. The linewidth of the laser is below the $10 \mathrm{kHz}$ level (integration time 0.1-1 s). Both L1(L2) and L3 are primarily intended for saturated subdoppler spectroscopy in iodine vapor at $532 \mathrm{~nm}$ wavelength and are designed to operate as laser optical frequency standards [32-38] with long-term frequency stability at $1 * 10^{-14}$ level. L4 laser is simple DPSSL (diode pumped solid state laser) with alignment-free monolithic resonator equipped with slow thermal frequency tuning option which allows the linear absorption spectroscopy frequency stabilization technique. Some results of the longterm frequency stability of this laser stabilized by linear absorption technique are in [20-22]. The denoted linewidth is below the $1 \mathrm{MHz}$ level and short-term optical power noise below $0.2 \%(10 \mathrm{~Hz}-20 \mathrm{MHz})$. L5 and L6 lasers are commonly used commercial single-mode He-Ne lasers from different manufacturers. L5 is equipped with internal thermal prestabilization by 2 -mode technique [10-12] with denoted relative frequency stability of $2 * 10^{-9} / 1 \mathrm{~min}$ integration time and amplitude noise below 5\%. L6 is a single-mode $\mathrm{He}-\mathrm{Ne}$ with denoted amplitude noise below $5 \%$ $(30 \mathrm{~Hz}-10 \mathrm{MHz}) . \mathrm{L} 7$ is an approximately 25 year old $\mathrm{He}-$ $\mathrm{Ne}$ laser, where worse properties due to degradation of the laser tube during long time period can be expected. Finally, L8 is a He-Ne- $\mathrm{I}_{2}$ laser - molecular iodine stabilized laser standard for $633 \mathrm{~nm}$ wavelength made at our institute about 10 years ago [48]. The interferometric measurement of the frequency noise was not done for L7 and L8 due to insufficient optical power available to feed both interferometer and the cavity. Correct operation of both frequency discriminators was evaluated by measurement of frequency modulated laser. L1 laser includes a tuning option through the mirror holder equipped with piezoelectric actuator (PZT). This PZT was modulated by sinusoidal signals of different frequencies $(1-30 \mathrm{kHz})$ and amplitudes, and results from cavity and also interferometer measurements were compared. The example of this measurement done for $13 \mathrm{kHz}$ modulation is in Fig.3., which shows good correlation between $1^{\text {st }}$ harmonics of modulation signal (differences on level of $2 \mathrm{~dB}(/ 1 \mathrm{MHz})$ ).

Amplitude noise measurements (range 0-100 kHz) were done for free-running lasers directly by measurement of optical power with the help of a low noise photodetector after few hours of stable operation of all the laser heads (Fig.4.). The best results were obtained with L1, 2 and L3 (both of them designed for frequency standards) with noise floor level below $-70 \mathrm{dBm},-62 \mathrm{dBm}$, respectively. L4 (simple DPSSL) shows LF fluctuations in the region of $0-10$ $\mathrm{kHz}$, caused probably by acoustic noise interference due to mechanical vibrations of the fan-cooled laser head. In case of He-Ne lasers the best result was obtained with L8 (He-
$\mathrm{Ne}-\mathrm{I}_{2}$ optical frequency laser standard), with noise floor at $65 \mathrm{dBm}$ and few harmonics (about $-55 \mathrm{dBm}$ ), especially in the region of $35 \mathrm{kHz}$. The rest of the $\mathrm{He}-\mathrm{Ne}$ lasers show amplitude fluctuations in range of -45 to $-50 \mathrm{dBm}$. We suggest that these fluctuations are mainly caused by the use of switched power-supplies and in case of L7 also by ageing of the old laser tube.

Table 1. A list of tested laser sources

\begin{tabular}{|c|c|c|c|}
\hline $\begin{array}{c}\text { Laser } \\
\text { no. }\end{array}$ & Description & $\begin{array}{c}\lambda \\
{[\mathbf{n m}]}\end{array}$ & $\begin{array}{c}\text { Optical } \\
\text { power } \\
{[\mathbf{m W}]}\end{array}$ \\
\hline L1 & $\begin{array}{c}\text { Nd:YAG external cav. doubling, } \\
\text { + noise-eater }\end{array}$ & 532 & 20 \\
\hline L2 & $\begin{array}{c}\text { Nd:YAG external cav. doubling, } \\
\text { - noise-eater }\end{array}$ & 532 & 20 \\
\hline L3 & Nd:YAG internal cav. doubling & 532 & 30 \\
\hline L4 & Nd:YAG, Simple DPSSL & 532 & 50 \\
\hline L5 & $\begin{array}{c}\text { He-Ne, general purposes, } \\
\text { thermally prestabilized }\end{array}$ & 633 & 5 \\
\hline L6 & He-Ne, general purposes & 633 & 0.8 \\
\hline L7 & He-Ne, 25 years old & 633 & 0.2 \\
\hline L8 & He-Ne-I2 standard, 633 nm & 633 & 0.2 \\
\hline
\end{tabular}
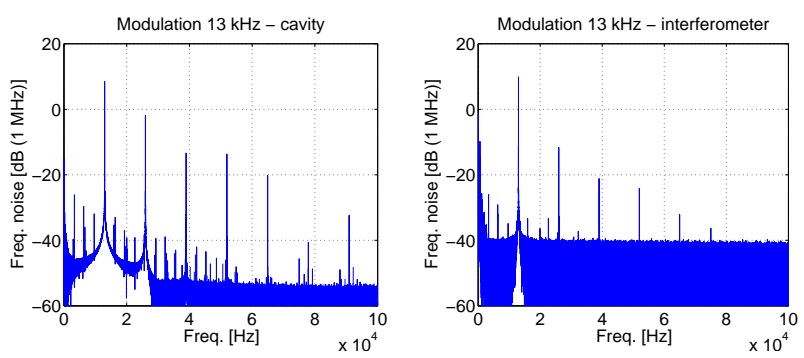

Fig.3. Test of proper operation of both frequency discriminators through introduction of frequency modulation by the tuning of the PZT of the laser L1.

The frequency noise measurements (Fig.5. for Nd:YAGs, Fig.6. for He-Nes) were done through the Fabry-Perot cavity and interferometer simultaneously to have the possibility of comparison of the results when the proper operation of both frequency discriminators was tested (Fig.3.).

To clarify the impact of the frequency noise of the laser on the interferometric measurement, we computed several values of the length noise caused by this source of uncertainty (Table 2.). When the $\Delta f$ is the RMS value of the frequency noise within the considered bandwidth, the corresponding RMS value of the length variations at the output of the interferometer ("length noise") is: 


$$
\Delta L=L_{D I F F} *\left(\frac{f_{0}}{f_{0}-\Delta f}-1\right)
$$

where $L_{D I F F}$ is length difference between reference and measuring paths of the interferometer and $f_{0}$ is the central optical frequency of the laser source $\left(f_{0}=c / \lambda_{0}\right)$.

Table 2. Computed RMS length noise values [m] in dependency on interferometer path distance difference and frequency noise of the laser.

\begin{tabular}{|c|c|c|c|c|}
\hline & \multicolumn{4}{|c|}{$\Delta \mathrm{f}[\mathrm{kHz}]$} \\
\hline $\mathrm{L}_{\text {DIFF }}[\mathrm{m}]$ & $1 \mathrm{e} 1$ & $1 \mathrm{e} 2$ & $1 \mathrm{e} 3$ & $1 \mathrm{e} 4$ \\
\hline 0.1 & $1.77 \mathrm{e}-12$ & $1.77 \mathrm{e}-12$ & $1.77 \mathrm{e}-10$ & $1.77 \mathrm{e}-9$ \\
\hline
\end{tabular}
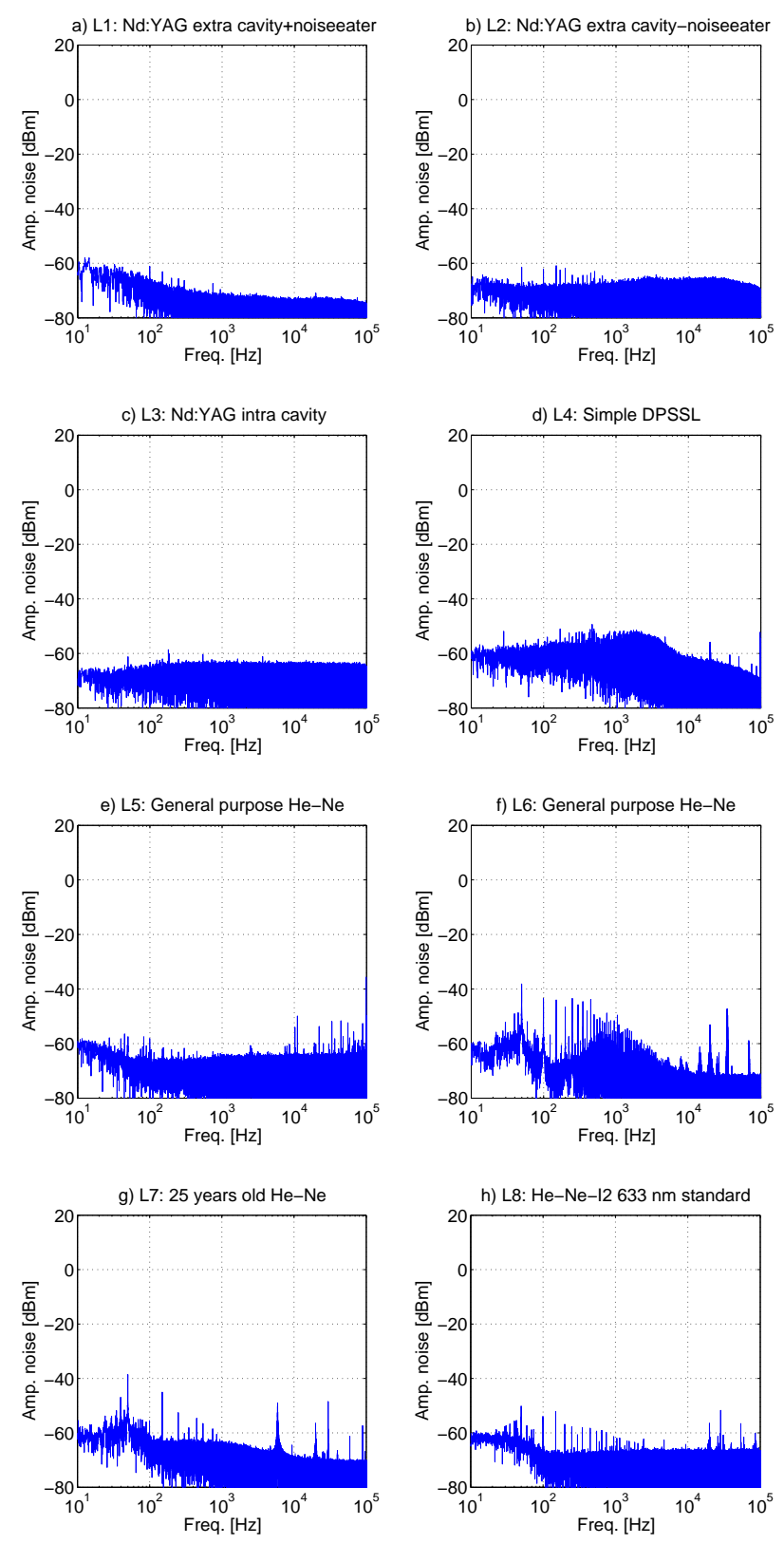

Fig.4. Measured amplitude noise of investigated lasers a)-h).
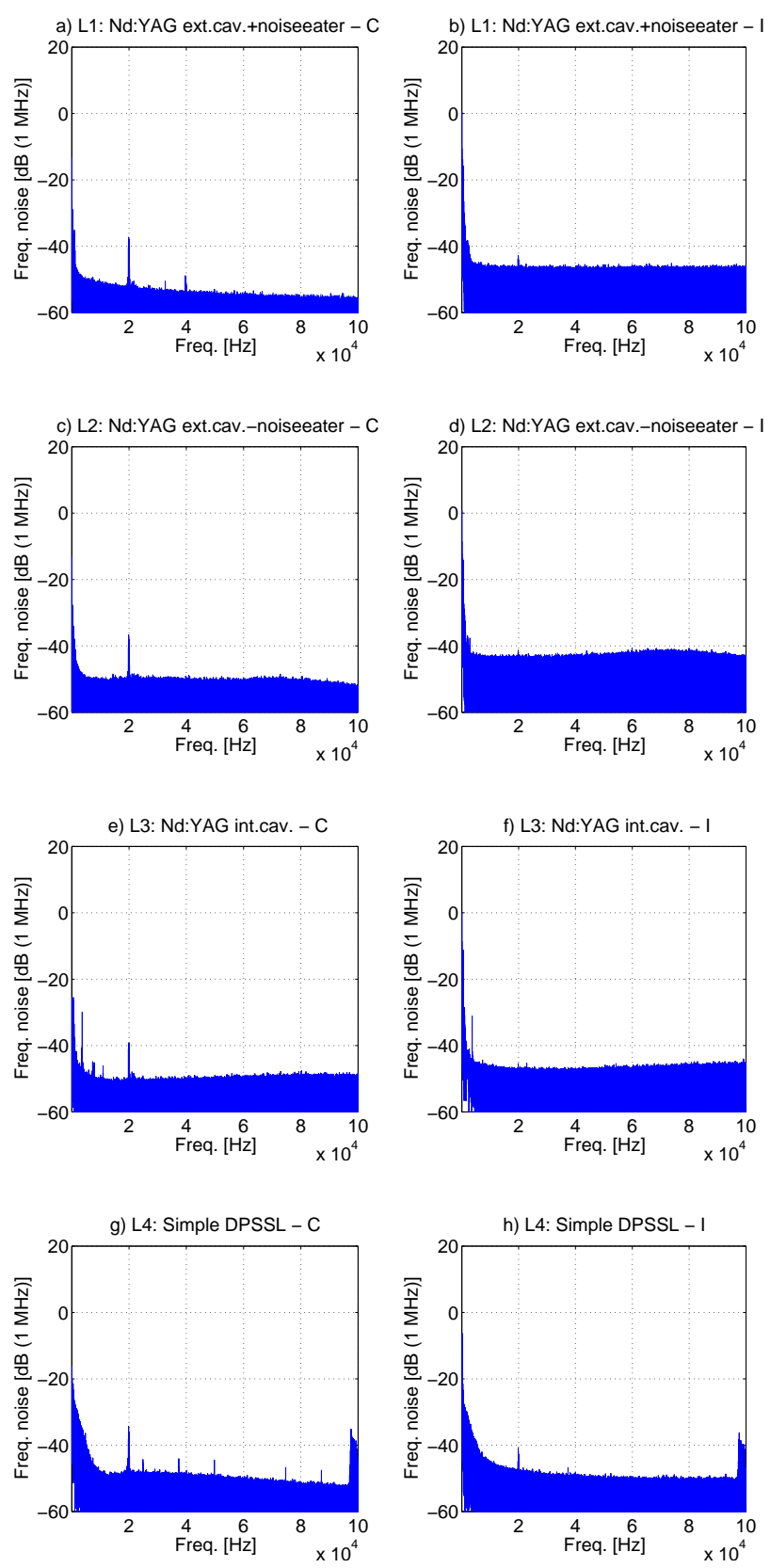

Fig.5. Measured frequency fluctuations of the investigated lasers Nd:YAG lasers (through Fabry-Perot cavity - suffix "C") and interferometer - suffix "I").

When we put together (1) and measured frequency noise from the Fabry-Perot cavity frequency discriminator, we can estimate the contribution of the laser frequency noise to the overall uncertainty of the interferometric length measurement. For the considered frequency bandwidth from $100 \mathrm{~Hz}$ to $300 \mathrm{kHz}$ and interferometric path length difference of $0.1 \mathrm{~m}$ we get RMS noise of the measured length of $8.83 \mathrm{e}-13 \mathrm{~m}$ for the laser L1 up to $5.99 \mathrm{e}-11 \mathrm{~m}$ length noise for the laser L7. So the frequency noise contribution to the measurement uncertainty should be considered with great care. 

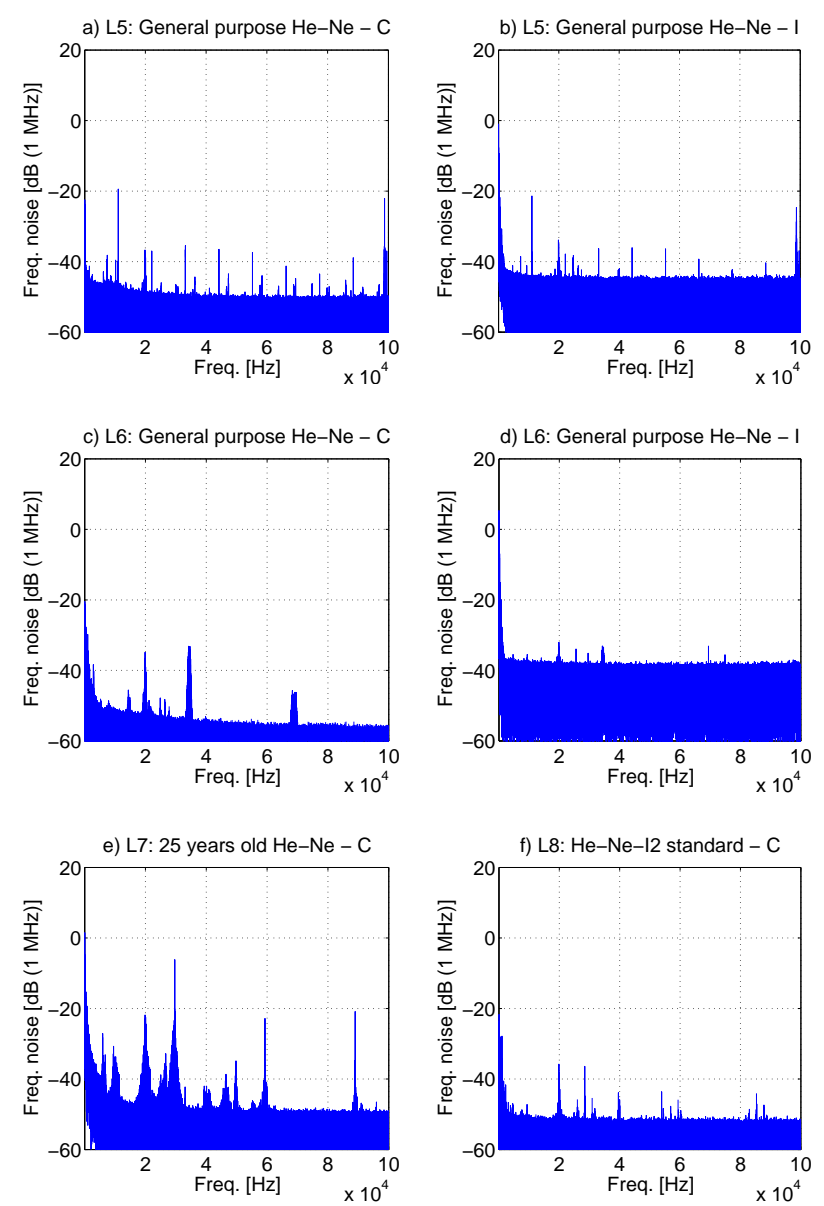

Fig.6. Measured frequency fluctuations of the investigated lasers He-Ne lasers (through Fabry-Perot cavity - suffix "C") and interferometer - suffix "I").

\section{CONCLUSION}

The amplitude and frequency noise of different laser sources intended for interferometry were measured. The experimental setup with two frequency discriminators was assembled. First discriminator was represented by FabryPerot cavity with length stabilized through the slow servoloop to the frequency of the investigated laser optical frequency, the second frequency discriminator was the Michelson interferometer. Measurements of amplitude and frequency noises were done simultaneously with the help of high-speed digitization card. The detection chain was not completely free of unwanted interfering signal with $20 \mathrm{kHz}$ frequency caused probably by some switched power supply in the powering network. It was not possible to identify this noise source and suppress its influence fully, so the frequency components of this interfering signal are present in the measurement spectra. The best results of the amplitude noise perform the $532 \mathrm{~nm} \mathrm{Nd:YAG} \mathrm{standards}$ (L1,2 and L3), in case of He-Ne and $633 \mathrm{~nm}$ wavelength the best laser is $\mathrm{L} 8$ (He-Ne- $\mathrm{I}_{2}$ standard). All of these lasers are intended to operate as laser standards for realization of fundamental etalons of length at 532 and $633 \mathrm{~nm}$, respectively, in the laboratory environment. Their design is more precise, these lasers are more expensive in comparison to other tested laser sources. The correct operation of frequency discriminators was tested with the help of additional modulation of the laser frequency. The results of frequency measurements show very good correlation between Fabry-Perot measurements and interferometer measurements. However, it can be seen that there are differences especially at low frequencies of spectrum recordings which are caused by insufficient vibration isolation and acoustic shielding of the interferometer. Next, it can be seen from the recordings that the noise floor as well as the resolution of the interferometer itself, are above the noise of tested lasers (for the set parameters - difference between interferometer reference and measuring beam paths and resolution of the detection systems). L4 (simple DPSSL) contains internal servo loops for driving the laser and also a fan for cooling of the laser which caused huge frequency variations in the low frequency range. The worst results of frequency stability shows the L7 laser (old one), mainly due to degradation due to the long history of this laser, old construction of the laser itself and its power supply. The next aspect is the resolution limit of the interferometer and its detection chain, which is higher than the noise of the investigated lasers (especially less noisy $\mathrm{Nd}$ :YAGs). We expect that greatest contributors to the frequency noise in the recordings are the switching power supplies of the laser heads. The results show better performance in frequency stability for measuring systems with $\mathrm{Nd}$ :YAG lasers in comparison to traditionally used $\mathrm{He}-\mathrm{Ne}$. This corresponds to measurements of long-term frequency stabilities which were done before [20-22]. Higher power available with Nd:YAG is not only high enough for feeding all of the interferometers but high power level incident on the photodetectors needs lower gain of the following amplifiers and thus improves the noise performance of the detection chain. Two of the last lasers (L7 and L8) have insufficient power for powering the interferometer and the Fabry-Perot cavity at once. The example values of the "length noise" at the output of the interferometer were calculated for the best and also the worst laser from the measured frequency noise results so the impact of this source of noise on the overall uncertainty of the interferometric system is shown.

\section{ACKNOWLEDGMENT}

The authors wish to express thanks for support to the grant projects from the Grant Agency of CR, projects: GPP102/11/P820 and GA102/09/1276, Academy of Sciences of CR, project: KAN311610701, Ministry of Education, Youth and Sports of CR, projects: CZ.1.05/2.1.00/01.0017 and LC06007, European Social Fund and National Budget of the Czech Republic, project: CZ.1.07/2.4.00/31.0016 and Technology Agency of CR, project: TA02010711.

\section{REFERENCES}

[1] Korpelainen, V., Seppa, J., Lassila, A. (2010). Design and characterization of MIKES metrological atomic force microscope. Precision Engineering, 34 (4), 735744.

[2] Otsuka, J., Ichikawa, S., Masuda, T., Suzuki, K. (2005). Development of a small ultraprecision positioning device with $5 \mathrm{~nm}$ resolution. Measurement Science and Technology, 16 (11), 2186-2192. 
[3] Dai, G.L., Pohlenz, F., Danzebrink, H.U., Xu, M., Hasche, K., Wilkening, G. (2004). Metrological large range scanning probe microscope. Review Scientific Instruments, 75 (4), 962-969.

[4] Lazar, J., Cip, O., Cizek, M., Hrabina, J., Sery, M., Klapetek, P. (2010). Interferometer controlled positioning for nanometrology. In Nanocon 2010 : 2nd International Conference, 12-14 October 2010, 287291.

[5] Kim, J.A., Kim, J.W., Park, B.C., Eom, T.B. (2006). Measurement of microscope calibration standards in nanometrology using a metrological atomic force microscope. Measurement Science and Technology, 17 (7), 1792-1800.

[6] Jäger, G., Gruenwald, R., Manske, E., Hausotte, T., Fuessl, R. (2004). A nanopositioning and nanomeasuring machine: Operation-measured results. Nanotechnology and Precision Engineering, 2, 81-84.

[7] Lazar, J., Hrabina, J., Sery, M., Klapetek, P., Cip, O. (2012). Multiaxis interferometric displacement measurement for local probe microscopy. Central European Journal of Physics, 10 (1), 225-231.

[8] Hrabina, J., Lazar, J., Klapetek, P., Cip, O. (2011). AFM nanometrology interferometric system with the compensation of angle errors. In Optical Measurement Systems for Industrial Inspection VII. SPIE, Vol. 8082, art. no. $80823 \mathrm{U}$.

[9] Quinn, T.J. (1994). Mise-en-pratique of the definition of the meter (1992). Metrologia, 30 (5), 523-541.

[10] Ciddor, P.E., Bruce, C.F. (1981). Long-term stability of a thermally-stabilized He-Ne laser. Metrologia, 17 (1), 17-18.

[11] Balhorn, R., Lebowsky, F., Kunzmann, H. (1972). Frequency stabilization of internal-mirror helium-neon lasers. Applied Optics, 11 (4), 742.

[12] Ciddor, P.E., Duffy, R.M. (1983). Two-mode frequency stabilized He-Ne (633 nm) lasers: Studies of short-term and long-term stability. Journal of Physics E, 16 (12), 1223-1227.

[13] Hrabina, J., Petru, F., Jedlicka, P., Cip, O., Lazar, J. (2007). Purity of iodine cells and optical frequency shift of iodine-stabilized He-Ne lasers. Journal of Optoelectronics and Advanced Materials, 1 (5), 202206.

[14] Sevcik, R., Guttenova, J. (2007). Primary length standard adjustment. In Wave and Quantum Aspects of Contemporary Optics : 15th Czech-Polish-Slovak Conference, 11-15 September 2007, 66-67.

[15] Bartl, J., Guttenova, J., Jacko, V., Sevcik, R. (2007). Circuits for optical frequency stabilization of metrological lasers. In Measurement 2007 : 6th International Conference on Measurement, 20-24 May 2007. IMS SAS, 131-134.

[16] Holzwarth, R., Nevsky, A.Y., Zimmermann, M. et al. (2001). Absolute frequency measurement of iodine lines with a femtosecond optical synthesizer. Applied Physics B, 73 (3), 269-271.
[17] Hrabina, J., Jedlicka, P., Lazar, J. (2008). Methods for measurement and verification of purity of iodine cells for laser frequency stabilization. Measurement Science Review, 8 (5), 118-121.

[18] Privalov, V.E., Shemanin, V.G., Voronina, E.I. (2010). Iodine molecules differential absorption cross section lidar studies. Measurement Science Review, 10 (3), 108-110.

[19] Simmons, J.D., Hougen, J.T. (1977). Atlas of I2 spectrum from 19000 to $18000 \mathrm{~cm}-1$. Journal of Research of the National Bureau of Standards A, 81 (1), 25-80.

[20] Hrabina, J., Lazar, J., Cip, O., Cizek, M. (2010). Laser source for interferometry in nanotechnology. In Wave and Quantum Aspects of Contemporary Optics : 17th Slovak-Czech-Polish Optical Conference, 6-10 September, 2010. SPIE, Vol. 7746, art. no. 77461I.

[21] Cao, H.J., Zang, E.J., Zhao, K., Zhang, X.B., Wu, Y.X., Shen, N.C. (1998). Frequency stabilization of a $\mathrm{Nd}$ : YAG laser to Doppler-broadened lines of iodine near 532nm. In Conference on Precision Electromagnetic Measurements Digest, 6-10 July 1998. IEEE, 183-184.

[22] Hrabina, J., Lazar, J., Klapetek, P., Cip, O. (2011). Multidimensional interferometric tool for the local probe microscopy nanometrology. Measurement Science and Technology, 22 (9), art. no. 094030.

[23] Lazar, J., Hola, M., Cip, O., Cizek, M., Hrabina, J., Buchta, Z. (2012). Displacement interferometry with stabilization of wavelength in air. Optics Express, 20 (25), 27830-27837.

[24] Edlen, B. (1966). The refractive index of air. Metrologia, 2, 71-80.

[25] Birch, K.P., Downs, M.J. (1994). Correction to the updated edlen equation for the refractive-index of air. Metrologia, 31 (4), 315-316.

[26] Lazar, J., Hola, M., Cip, O., Cizek, M., Hrabina, J., Buchta, Z. (2012). Refractive index compensation in over-determined interferometric system. Sensors, 12 (10), 14084-14094.

[27] Quoc, T.B., Ishige, M., Ohkubo, Y., Aketagawa, M. (2009). Measurement of air-refractive-index fluctuation from laser frequency shift with uncertainty of order 10(-9). Measurement Science and Technology, 20 (12), art. no. 125302.

[28] Lazar, J., Cip, O., Cizek, M., Hrabina, J., Buchta, Z. (2011). Standing wave interferometer with stabilization of wavelength on air. tm - Technisches Messen, 78 (11), 484-488.

[29] Zhang, J., Lu, Z.H., Menegozzi, B., Wang, L.J. (2006). Application of frequency combs in the measurement of the refractive index of air. Review Scientific Instruments, 77 (8), art. no. 083104.

[30] Lazar, J., Cip, O., Cizek, M., Hrabina, J., Buchta, Z. (2011). Suppression of air refractive index variations in high-resolution interferometry. Sensors, 11 (8), 7644-7655. 
[31] Lazar, J., Cip, O., Cizek, M., Hrabina, J., Buchta, Z. (2010). Interferometry with direct compensation of fluctuations of refractive index of air. In Wave and Quantum Aspects of Contemporary Optics : 17th Slovak-Czech-Polish Optical Conference, 6-10 September, 2010. SPIE, Vol. 7746, art. no. 77460E.

[32] Nyholm, K., Merimaa, M., Ahola, T., Lassila, A. (2003). Frequency stabilization of a diode-pumped $\mathrm{Nd}$ :Yag laser at $532 \mathrm{~nm}$ to iodine by using thirdharmonic technique. IEEE Transaction on Instrumentation and Measurement, 52 (2), 284-287.

[33] Nevsky, A.Y., Holzwarth, R., Reichert, J. et al. (2001). Frequency comparison and absolute frequency measurement of I-2-stabilized lasers at $532 \mathrm{~nm}$. Optics Commumications, 192 (3-6), 263-272.

[34] Rovera, G.D., Ducos, F., Zondy, J.J., Acef, O., Wallerand, J.P., Knight, J.C., Russell, P.S. (2002). Absolute frequency measurement of an I-2 stabilized Nd:YAG optical frequency standard. Measurement Science and Technology, 13 (6), 918-922.

[35] Galzerano, G., Bava, E., Bisi, M., Bertinetto, F., Svelto, C. (1998). Frequency stabilization of frequency-doubled $\mathrm{Nd}$ : YAG lasers at $532 \mathrm{~nm}$. In Conference on Precision Electromagnetic Measurements Digest, 6-10 July 1998. IEEE, 193-194.

[36] Galzerano, G., Bava, E., Bisi, M., Bertinetto, F., Svelto, C. (1999). Frequency stabilization of frequency-doubled $\mathrm{Nd}$ : YAG lasers at $532 \mathrm{~nm}$ by frequency modulation spectroscopy technique. IEEE Transaction on Instrumentation and Measurement, 48 (2), 540-543.

[37] Lazar, J., Hrabina, J., Jedlicka, P., Cip, O. (2009). Absolute frequency shifts of iodine cells for laser stabilization. Metrologia, 46 (5), 450-456.

[38] Picard, S., Robertsson, L., Ma, L.S., Nyholm, K. et al. (2003). Comparison of ${ }^{127}$ I2-stabilized frequencydoubled Nd:YAG lasers at the Bureau International des Poids et Mesures. Applied Optics, 42 (6), 1019 1028 .
[39] Lance, A.L., Seal, W.D., Labaar, F. (1982). Phase noise measurement systems. ISA Transactions, 21 (4), 37-44.

[40] Badami, V.G., Patterson, S.R. (2000). A frequency domain method for the measurement of nonlinearity in heterodyne interferometry. Precision Engineering, 24 (1), 41-49.

[41] Cip, O., Petru, F. (2000). A scale-linearization method for precise laser interferometry. Measurement Science and Technology, 11 (2), 133-141.

[42] Eom, T., Kim, J., Jeong, K. (2001). The dynamic compensation of nonlinearity in a homodyne laser interferometer. Measurement Science and Technology, 12 (10), 1734-1738.

[43] Rerucha, S., Buchta, Z., Sarbort, M., Lazar, J., Cip, O. (2012). Detection of interference phase by digital computation of quadrature signals in homodyne laser interferometry. Sensors, 12 (10), 14095-14112.

[44] Siffalovic, P., Vegso, K., Jergel, M., Majkova, E. et al. (2010). Measurement of nanopatterned surfaces by real and reciprocal space techniques. Measurement Science Review, 10 (5), 153-156.

[45] Petru, F., Cip, O. (1999). Problems regarding linearity of data of a laser interferometer with a singlefrequency laser. Precision Engineering, 23 (1), 39-50.

[46] Smid, R., Cip, O., Lazar, J. (2008). Precise length etalon controlled by stabilized frequency comb. Measurement Science Review, 8 (5), 114-117.

[47] Meiners-Hagen, K., Schodel, R., Pollinger, F., AbouZeid, A. (2009). Multi-wavelength interferometry for length measurements using diode lasers. Measurement Science Review, 9 (1), 16-26.

[48] Petru, F., Popela, B., Vesela, Z. (1993). Design and performance of compact iodine stabilized He-Ne lasers at lambda $=633 \mathrm{~nm}$ with a short optical resonator. Measurement Science and Technology, 4 (4), 506-512.

Received October 2, 2012 Accepted April 4, 2013. 